\title{
Synergistic anti-glioma effect of a coloaded nano-drug delivery system
}

\author{
This article was published in the following Dove Press journal: \\ International Journal of Nanomedicine \\ 16 December 2016 \\ Number of times this article has been viewed
}

Huae $\mathrm{Xu}^{\mathrm{l}, *}$

Feng Jia ${ }^{2, *}$

Pankaj Kumar Singh ${ }^{3}$

Shu Ruan ${ }^{4}$

Hao Zhang,*

Xiaolin $\mathrm{Li}^{5}$

'Department of Pharmacy, The First Affiliated Hospital with Nanjing

Medical University, Nanjing,

${ }^{2}$ Department of Neurosurgery, Yancheng City No I People's Hospital,

The Fourth Affiliated Hospital of

Nantong Medical College, Yancheng,

People's Republic of China;

${ }^{3}$ Department of Experimental

Radiation Oncology, The University

of Texas MD Anderson Cancer Center,

Houston, TX, USA; ${ }^{4}$ Department

of Endocrinology, Yancheng Third

Hospital, The Affiliated Hospital of

Southeast University Medical College,

Yancheng, ${ }^{5}$ Department of Geriatrics,

The First Affiliated Hospital with

Nanjing Medical University, Nanjing,

People's Republic of China

*These authors contributed equally to this work

Correspondence: Shu Ruan

Department of Endocrinology, Yancheng

Third Hospital, The Affiliated Hospital

of Southeast University Medical College,

75 Juchang Road, Yancheng, People's

Republic of China

Tel +865I581606102

Fax +8681588303100

Email ycsrshuruan@I63.com

Xiaolin Li

Department of Geriatrics, The First

Affiliated Hospital with Nanjing Medical

University, 300 Guangzhou Road,

Nanjing, People's Republic of China

Tel +86 2568135151

Fax +862568I3 5I5I

Email |x|@njmu.edu.cn
Abstract: The anti-glioma effect of temozolomide (Tem) is sometimes undermined by the emerging resistance. Recently, resveratrol (Res), herbal medicine extracted from grape seeds, has been demonstrated for its potential use in chemosensitization. In the current study, both these drugs were loaded simultaneously into nanoparticles with methoxy poly(ethylene glycol)poly epsilon caprolactone (mPEG-PCL) as drug carriers in order to achieve better antitumor efficiency. Tem/Res-coloaded mPEG-PCL nanoparticles were constructed, characterized, and tested for antitumor effect on glioma cells by using in vitro and xenograft model system. The nanoparticle constructs were satisfactory with drug loading content (Res $=\sim 12.4 \%$; Tem $=\sim 9.3 \%$ ) and encapsulation capacity of $>85 \%$ for both the drugs. In addition, the coencapsulation led to better in vitro stability of the nanoparticles than Tem-loaded nanoparticles. An in vitro uptake study demonstrated a high uptake efficiency of the nanoparticles by glioma cells. The synergistic antitumor effect against glioma cells was observed in the combinational treatment of Res and Tem. Tem/Res-coloaded nanoparticles induced higher apoptosis in U87 glioma cells as compared to cells treated by the combination of free drugs. Tem/Res-coloaded particles caused more effective inhibition of phosphor-Akt, leading to upregulation of the downstream apoptotic proteins. In addition, the in vivo study showed the superior tumor delaying effect of coloaded nanoparticles than that of free drug combination. These results suggest that Tem/Res-coloaded nanoparticles could be a potential useful chemotherapeutic formulation for glioma therapy.

Keywords: resveratrol, temozolomide, synergy, mPEG-PCL, polymeric, drug delivery

\section{Introduction}

Temozolomide (Tem) has been used as the main chemotherapeutics for malignant glioma for decades, but its clinical application is hampered by the emerging resistance, which subsequently leads to recurrence of primary tumor. ${ }^{1}$ Moreover, unavoidable side effects of Tem, including nausea, vomiting, loss of appetite, diarrhea, skin rash, hair loss, tiredness, dizziness, blurred vision, insomnia, and headache, also constrain its clinical use. ${ }^{2}$ Therefore, there is a pressing need to develop a strategy to improve the efficacy and reduce the toxicity of Tem.

Herbal medicines have been reported for their potential in the therapy of many cancers. ${ }^{3}$ Resveratrol (3,5, $4^{\prime}$-trihydroxy-trans-stilbene) has been proved to be effective in inhibiting the growth of several types of tumor, such as colon, breast, pancreas, prostate, ovarian, and endometrial cancers, as well as lymphoma. ${ }^{4-7}$

Some previous studies also demonstrated the role of resveratrol (Res) in sensitizing tumor cells to the conventional chemotherapy, including enhancing the cytotoxicity of Tem against glioma cells. ${ }^{8-13}$ However, hydrophobicity and poor solubility of Res severely limit its clinical application. 
Nano drug delivery systems have been used to overcome the potential disadvantages of Res and Tem and to further improvise the delivery efficiency of both the drugs. ${ }^{14-17}$ Previous studies have demonstrated the superiority of drug-loaded nanoparticles with amphiphilic copolymer as drug carriers (eg, methoxy poly(ethylene glycol)-poly epsilon caprolactone [mPEG-PCL]). ${ }^{18-22}$ The hydrophobic part of the copolymers (PCL) is effective to encapsulate the insoluble drugs as the inner core of the nanoparticles, while the hydrophilic part (PEG) could be the outer shell with the capability to help the nanoparticle escape from the scavenge of reticuloendothelial system. ${ }^{23}$ Moreover, previous studies have demonstrated the effectiveness of delivering multiple drugs simultaneously, which provides the possibility to codeliver Res and Tem in amphiphilic copolymer nanoparticles. ${ }^{19}$ Most importantly, the synergistic antitumor effect of Res and Tem could also be achieved when they are delivered simultaneously by nanoparticles.

In the current study, Res and Tem were coencapsulated into mPEG-PCL nanoparticles. Then, the Tem/Res-coloaded nanoparticles (T/R-NPs) were characterized for the particle size and zeta potential. Cellular uptake study was conducted in order to evaluate the uptake efficiency by glioma cells. Antitumor effect was evaluated in glioma cells and xenograft model of nude mice. The purpose of the current study was to provide a feasible strategy to enhance the antiglioma effect of Tem by administering Res simultaneously in a nanoparticlebased drug delivery system.

\section{Materials and methods Materials}

Res was purchased from Sigma Chemical Co. (St Louis, MO, USA). Tem was a gift from Tasly Pharmaceutical Co. Ltd. (Tianjin, People's Republic of China). mPEG (molecular weight $[\mathrm{MW}]=4 \mathrm{kDa}$ ) and $\varepsilon$-caprolactone were purchased from Sigma Chemical Co. 2,3-Bis-(2-Methoxy-4-Nitro-5Sulfophenyl)-2H-Tetrazolium-5-Carboxanilide (XTT) was a product of Roche Company. Coumarin-6 was purchased from Sigma Chemical Co. Primary antibodies (ie, anti-p-Akt, antiAkt, anti-Bax, anti-Bcl-2, and anti-Caspase-3) and secondary antibodies were purchased from Cell Signaling Technology. Anti-actin was purchased from Sigma Chemical Co. All other reagents used were of analytical grade.

\section{Methods}

Synthesis of mPEG-PCL block copolymer and preparation of T/R-NPs

mPEG4k-PCL20k block copolymers were synthesized as reported in some previous studies. ${ }^{20,21} \mathrm{~T} / \mathrm{R}-\mathrm{NPs}$ were prepared by a nanoprecipitation method with different feeding ratios as described previously with some modifications. ${ }^{19}$ For example, in 1:1 feeding ratio, $5 \mathrm{mg}$ Res and $30 \mathrm{mg}$ polymer powder were dissolved in $500 \mu \mathrm{L}$ acetone; $5 \mathrm{mg}$ Tem was dissolved in $10 \mathrm{~mL}$ water $\left(55^{\circ} \mathrm{C}\right)$. Then, the organic phase of $500 \mu \mathrm{L}$ Res and polymer solution in acetone was added drop-wise into $10 \mathrm{~mL}$ Tem solution in water. The solution was placed into a dialysis bag (molecular weight cutoff $[\mathrm{MWCO}]=12,000 \mathrm{Da}$ ) in order to remove acetone thoroughly. After 4-h dialysis of changing outside medium for every $30 \mathrm{~min}$, the solution was filtered through a $220-\mathrm{nm}$ filter to remove unloaded drugs and polymer aggregates. Fluorescent nanoparticles with coumarin- 6 as a fluorescent marker were prepared by adding coumarin- 6 and Res together into the acetone. Then, T/R-NPs solution was lyophilized with poloxamer 188 as a cryoprotectant for in vitro and in vivo use.

\section{Characterization of T/R-NPs}

Mean diameter and size distribution of nanoparticles before lyophilization were dissolved in double-distilled water and measured by dynamic light scattering using a Brookhaven BI-9000AT instrument (Brookhaven Instruments Corporation, Holtsville, NY, USA). Zeta potential of nanoparticles was measured by the laser Doppler anemometry (Zeta Plus, Zeta Potential Analyzer; Brookhaven Instruments Corporation).

\section{Drug loading efficiency}

The concentrations of Res and Tem were measured by using high-performance liquid chromatography (HPLC) on a Shimadzu LC-10AD (Shimadzu, Japan) with an ultraviolet (UV) detector and an Agilent C-18 analytical column. ${ }^{7,24}$ The mobile phase for Tem was methanol/ $0.5 \%$ acetic acid $(20: 80, \mathrm{v} / \mathrm{v})$. The pump rate was $1.1 \mathrm{~mL} / \mathrm{min}$, and the retention time was $3.682 \mathrm{~min}$. The wavelength of $330 \mathrm{~nm}$ was used to determine the concentration of Tem. The mobile phase for Res consisted of methanol (spectral grade, Merck, Germany)/ double-distilled water/glacial acetic acid (48/52/0.05, v/v/v) and was pumped at a flow rate of $1.0 \mathrm{~mL} / \mathrm{min}$ with the retention time of $5.855 \mathrm{~min}$ at a wavelength of $303 \mathrm{~nm}$. The concentration of each drug was calculated based on the peak area with reference to a calibration curve.

The drug loading content and encapsulation efficiency of the nanoparticles were calculated as follows:

$$
\begin{aligned}
& \begin{array}{l}
\text { Drug loading } \\
\text { content (\%) }
\end{array}=\frac{\text { Weight of the drug in nanoparticles }}{\text { Weight of the nanoparticles }} \times 100 \% \\
& \begin{array}{l}
\text { Encapsulation } \\
\text { efficiency (\%) }
\end{array}=\frac{\text { Weight of the drug in nanoparticles }}{\text { Weight of the feeding drugs }} \times 100 \%
\end{aligned}
$$




\section{In vitro release study}

About $10 \mathrm{mg}$ T/R-NPs lyophilized powder was dissolved in $1 \mathrm{~mL}$ phosphate-buffered saline (PBS) and placed inside a dialysis bag ( $\mathrm{MWCO}=12 \mathrm{kD}$ ). The bag was immersed in $50 \mathrm{~mL}$ PBS with slight stirring. At each time point, $1 \mathrm{~mL}$ solution from the dialysis bag was withdrawn and measured for the concentration of Res and Tem by using HPLC. The same amount of PBS was added back to the outside medium at the same time of sampling. Sink condition was maintained in the whole experiment.

\section{Cellular uptake study}

Cellular uptake by glioma cells was evaluated through confocal laser scanning microscopy (CLSM) and fluorescent microscopy with coumarin- 6 as a fluorescent marker. Briefly, human malignant glioma cell line U87, obtained from Shanghai Institute of Cell Biology (Shanghai, People's Republic of China), cells were seeded in 6-well plates and allowed to adhere for $24 \mathrm{~h}$. Coumarin-6-loaded T/R-NPs were incubated with U87 cells for different time periods (ie, 30, 60, 120, and $180 \mathrm{~min}$ ). At the end of incubation, cells were washed and then observed under CLSM and fluorescent microscopy. For the quantification study, cells were washed three times and centrifuged at a speed of $1,500 \mathrm{rpm}$ for $5 \mathrm{~min}$. Then, the cell pellets were dissolved in $1 \mathrm{~mL}$ methanol, vortexed, and sonicated. The cell suspension was centrifuged at a speed of 10,000 rpm, and the supernatant was transferred to an Eppendorf tube. Then, the supernatants were detected by using a fluorescent spectrometer at an excitation wavelength of $458 \mathrm{~nm}$ and an emission wavelength of $540 \mathrm{~nm}$.

\section{In vitro cytotoxicity and isobologram analysis}

The synergy of both the drugs was analyzed by using isobologram analysis as reported previously. ${ }^{25}$ Cytotoxicity of each drug was determined by XTT assay. Cells were seeded in 96-well plates with a density of 5,000 cells/well. Cells were allowed to adhere for $24 \mathrm{~h}$ to form monolayer. Then, the concentration of Res was fixed at 2 and $4 \mu \mathrm{g} / \mathrm{mL}$ and Tem was treated at a series of concentrations (ie, 4, 8, 16, 32,64 , and $128 \mu \mathrm{g} / \mathrm{mL}$ ). For the XTT assay, U87 cells were exposed to different concentrations of Res and Tem alone or in combination for $48 \mathrm{~h}$. After treatment, media containing drugs were discarded, and fresh medium with XTT was added into the wells. Absorptance was measured after $18 \mathrm{~h}$ by using a UV/visible spectrometer with a wavelength of $490 \mathrm{~nm}$.

Combination index (CI) was calculated by using the following equation:

$$
\mathrm{CI}=\left(\mathrm{dR} / \mathrm{D}_{50} \mathrm{R}\right)+\left(\mathrm{dT} / \mathrm{D}_{50} \mathrm{~T}\right)
$$

$D_{50} R$ is the concentration of Res which can produce $50 \%$ of cell death alone, whereas $\mathrm{dR}$ is the concentration of Res which leads to $50 \%$ cell inhibition with the combination of Tem. $\mathrm{D}_{50} \mathrm{~T}$ is the concentration of Tem which can produce $50 \%$ of cell death alone, whereas dT is the concentration of Tem which leads to $50 \%$ cell inhibition with the combination of Res. CI value $<1$ is defined as synergism, and CI value $>1$ is defined as antagonism. The addition is defined when the $\mathrm{CI}$ value $=1$.

The concentration used in in vitro studies was according to the isobologram results and was achieved by the mixture of T/R-NPs with different feeding ratios.

\section{Apoptotic induction}

To quantify the apoptosis rate, U87 cells were treated with different agents and stained with annexin V-fluorescein isothiocyanate (FITC) and propidium iodide by using the annexin V-FITC kit (eBioscience Inc., San Diego, CA, USA). Then, the cells were analyzed by using FACScan flow cytometer (Becton, Dickinson and Company, Franklin Lakes, NJ, USA).

\section{Protein expression}

U87 cells were treated with either singly or in a combination of Res and Tem, as well as codrug loaded nanoparticles (T/R-NPs) for $6 \mathrm{~h}$. The concentrations of Res and Tem were 4 and $12 \mu \mathrm{g} / \mathrm{mL}$, respectively. Protein expressions were determined by Western blot as reported previously. ${ }^{18}$ The primary antibodies were anti-p-Akt, anti-Akt, antiBcl-2, anti-Bax, and anti-actin. The secondary antibodies were selected according to the origin of primary antibodies. Bands were analyzed by using Densitometric Image Analysis Software (Quantity One, Bio-Rad, Hercules, CA, USA).

\section{Wound healing assay}

Cell migration was evaluated by wound healing assay as described previously. ${ }^{18}$ Briefly, U87 cells were seeded in 6-well plates and allowed to grow for at least $24 \mathrm{~h}$ to $80 \%-90 \%$ confluent. After cells were serum-starved for $12 \mathrm{~h}$, a vertical clear space was made by the scratch of pipette tip, and the cells were pictured at this time point (designated as $0 \mathrm{~h}$ ). After $24 \mathrm{~h}$ of treatments of different agents, cells were photographed again in the same field. Then, the pictures were superposed in Photoshop (Adobe Systems Incorporated, San Jose, CA, USA), and areas were measured by using Scion Image Analysis Software (Scion Corporation, Frederick, MD, USA). The cell migration rate was calculated according to the following formula: (the average area of the wound at $0 \mathrm{~h}$ - the average area of the wound at 24 or $48 \mathrm{~h}$ )/the average area of the wound in $0 \mathrm{~h}$. 


\section{In vivo study}

Nude mice (aged 6-8 weeks) weighing 18-20 g were purchased from the Animal Experiment Center of Nanjing Medical University. The mice were reared under specific pathogen-free circumstances. The in vivo animal studies were executed in full compliance with the ARRIVE guidelines (Animal Research: Reporting In Vivo Experiments) for cancer study approved by the Animal Care Committee at Nanjing Medical University (Nanjing, People's Republic of China). U87 cell suspension $(100 \mu \mathrm{L})$ at a density of $1.5 \times 10^{6}$ cells were injected subcutaneously into the left axillary space of the mice. When the tumor grew to a size of $70-80 \mathrm{~mm}^{3}$ after 10-14 days, the mice were treated with different agents intraperitoneally (IP). The dose of Res was $10 \mathrm{mg} / \mathrm{kg}$, whereas the dose of Tem was $30 \mathrm{mg} / \mathrm{kg}$. T/R-NPs were administered at the equivalent doses of free Res and Tem. All the mice were ear-tagged. Tumor volumes were measured every other day during the 2-week experiment. Tumor volume was calculated by using the equation, $\left(W^{2} \times L\right) / 2$, where $W$ is the tumor measurement at the widest point, and $L$ is the tumor dimension at the longest point. Relative tumor volume was calculated by the formula $\left(V_{\mathrm{n}} / V_{0}\right)$, where $V_{\mathrm{n}}$ is the tumor volume measured at the corresponding day, and $V_{0}$ is the tumor volume measured at Day 0. Mice were weighed before the drug treatment

A

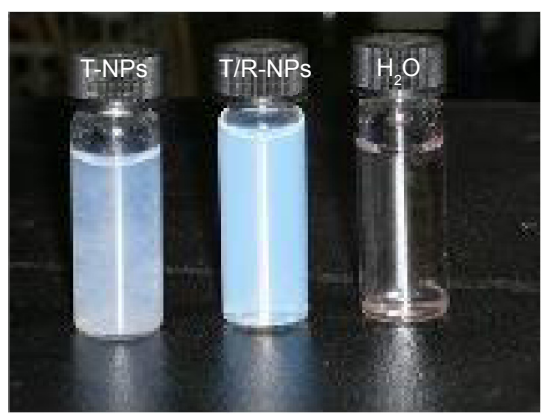

C

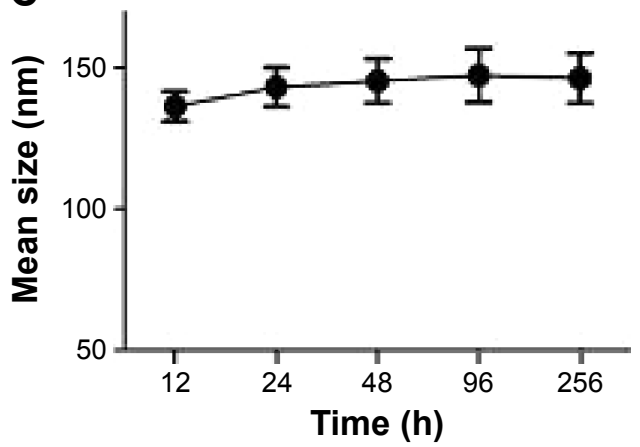

so that the dosage could be adjusted to achieve the $\mathrm{mg} / \mathrm{kg}$ amounts reported. Animals were also weighed thrice a week (on alternate days) throughout the experiment.

\section{Statistical analysis}

All the data in this manuscript are expressed as mean \pm standard deviation of three independent experiments. Statistical comparisons of relative groups were based on Student's $t$-test or one-way analysis of variance with Statistical Package for Social Sciences software, Version 11.5 (IBM Corporation, Armonk, NY, USA). The data were considered significant at $P=0.05$.

\section{Results}

\section{Characterization, loading efficiency, and in vitro release of T/R-NPs}

As shown in Figure S1, the solubility of Tem was very poor in regular water $\left(20^{\circ} \mathrm{C}-30^{\circ} \mathrm{C}\right)$ at a concentration of $250-630 \mu \mathrm{g} / \mathrm{mL}$, whereas it increased greatly to $>3 \mathrm{mg} / \mathrm{mL}$ in hot water $\left(50^{\circ} \mathrm{C}\right.$; Figure S1), which made it possible for nanoparticle encapsulation. However, as shown in Figure 1A, T-NPs were extremely unstable during the preparation process. It precipitated immediately after the organic phase was removed by dialysis. In contrast, coencapsulation of Res in Tem-loaded mPEG-PCL nanoparticles greatly increased
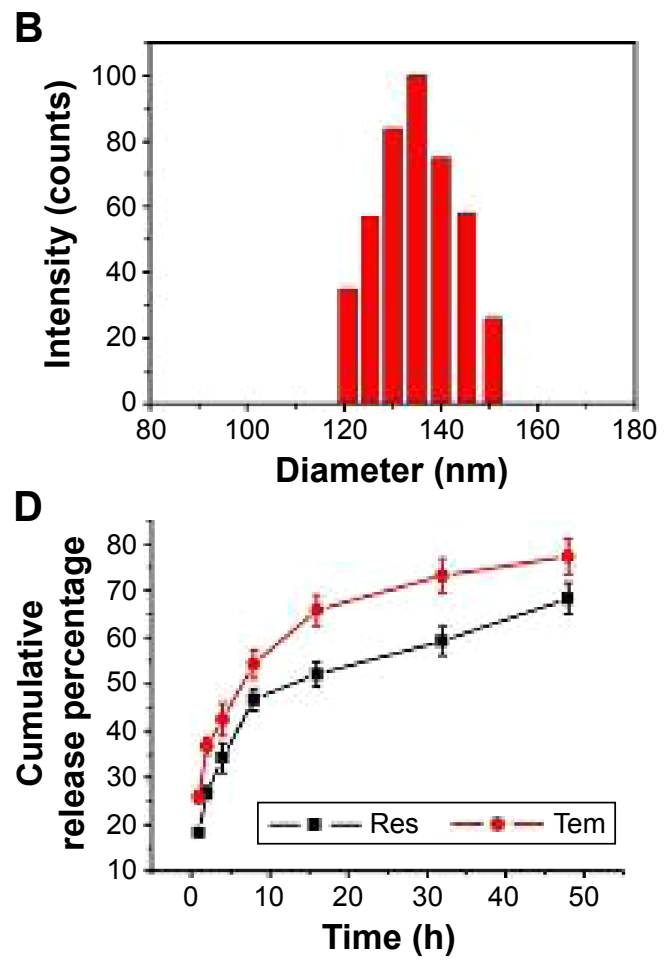

Figure I Characterization of T/R-NPs.

Notes: (A) Images of T-NPs (left), T/R-NPs (middle), and water (right). (B) Size distribution of T/R-NPs through DLS. (C) Stability test of T/R-NPs at room temperature. (D) In vitro release curves of T/R-NPs.

Abbreviations: DLC, drug loading content; Res, resveratrol; Tem, temozolomide; T/R-NPs, Tem/Res-coloaded nanoparticles; h, hours. 
Table I Characterization and drug loading efficiency of T/R-NPs

\begin{tabular}{llllll}
\hline Nanoparticle & Size $(\mathbf{n m})^{\mathbf{a}}$ & Polydispersity & Zeta potential $(\mathbf{m V})$ & DLC & EE \\
\hline Empty NP & $130.3 \pm 7.3$ & 0.14 & $-4.3 \pm 0.4$ & - & - \\
T/R-NP & $135.3 \pm 5.6$ & 0.14 & $-3.4 \pm 0.6$ & $12.4 \% \pm 2.3 \%$ (Res) & $83.2 \% \pm 5.1 \%($ Res $)$ \\
& & & & $9.3 \% \pm 1.5 \%($ Tem) & $85.2 \% \pm 3.5 \%($ Tem) \\
\hline
\end{tabular}

Note: aStandard deviation was calculated for the mean particle size obtained from the three measurements of a single batch.

Abbreviations: DLC, drug loading content; EE, encapsulation efficiency; Res, resveratrol; Tem, temozolomide; T/R-NPs, Tem/Res-coloaded nanoparticles.

the stability of the nanoparticles, which was limpid and bluish solution with no aggregates.

DLS indicated that the size of T-NPs was $>10 \mu \mathrm{m}$, whereas the average size of T/R-NPs with a feeding ratio of $1: 1$ was $\sim 135.3 \pm 5.6 \mathrm{~nm}$ (Table 1 and Figure 1B). Zeta potential of T/R-NPs was slightly negative with a value of $-3.4 \pm 0.6 \mathrm{mV}$, which was similar to that of empty NPs (Table 1). Moreover, T/R-NPs were very stable at a room temperature during the following 8 days after they were made (Figure 1C).

Figure S2 shows the data from HPLC of both Res and Tem. The retention times for Tem and Res, respectively, were 3.682 and $5.855 \mathrm{~min}$ with no overlap, making it feasible for detection. As shown in Table 1, by varying the feeding ratio of each drug and the polymers, the highest drug loading content was $12.4 \% \pm 2.3 \%$ (Res) and $9.3 \% \pm 1.5 \%$ (Tem) at the feeding ratio of $1: 1$, respectively (Figure S3), with the encapsulation efficiency of both the drugs being $>80 \%$. Figure 1D shows an in vitro release pattern for each drug. Both the drugs were released from the nanoparticles in a sustained manner. There was a burst release of each drug during the first several hours, followed by a controlled release in the rest period.

\section{Evaluation of cellular uptake efficiency of T/R-NPs by glioma cells}

A lipophilic dye, coumarin-6, was coloaded into T/R-NPs and used as a nanoparticle tracer. The upper and central panels of Figure 2 were the CLSM and a fluorescent image of glioma
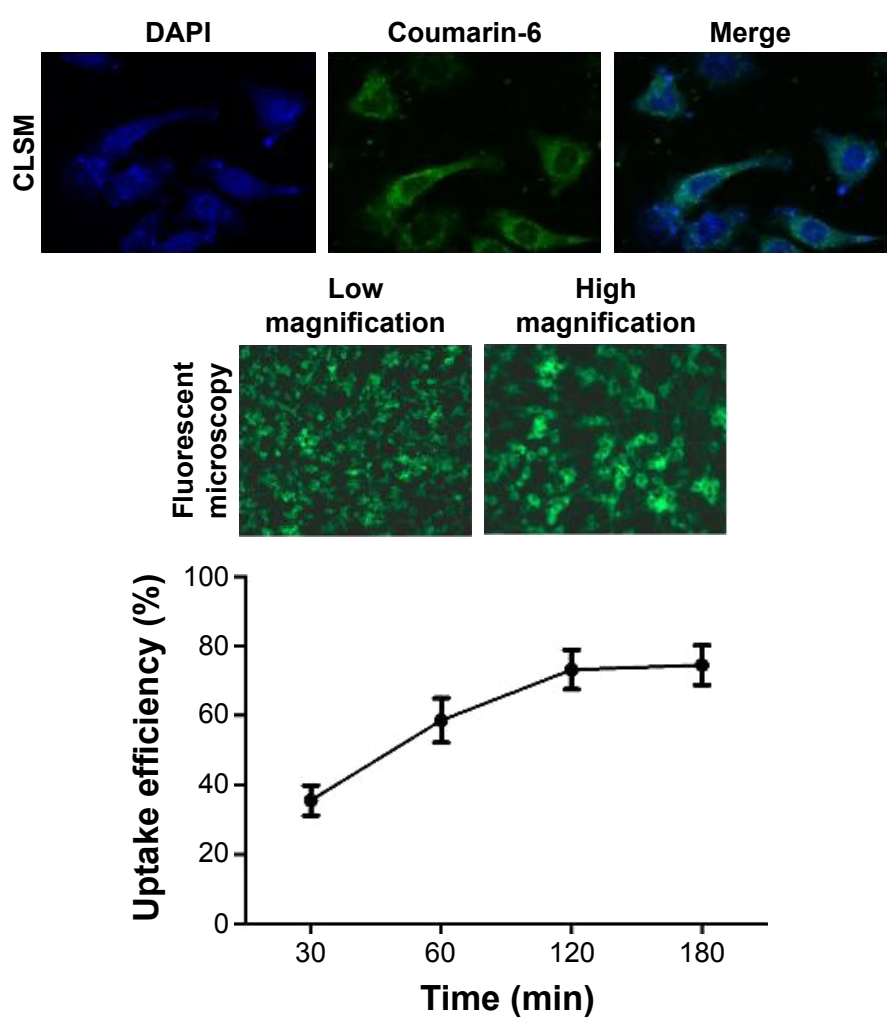

Figure 2 Cellular uptake of T/R-NPs by U87 cells.

Notes: Top panel: CLSM image of cells incubated with coumarin-6-loaded T/R-NPs (DAPI-Blue; coumarin-6-Green). Middle panel: fluorescent microscopy of cells incubated with coumarin-6-loaded T/R-NPs. Bottom panel: quantification of cellular uptake.

Abbreviations: CLSM, confocal laser scanning microscopy; DAPI, 4',6-diamidino-2-phenylindole; DLC, drug loading content; Res, resveratrol; Tem, temozolomide; T/R-NPs, Tem/Res-coloaded nanoparticles; min, minutes. 
cells incubated with T/R-NPs for 2 h, respectively. Green fluorescence generated by coumarin- 6 indicated the cellular distribution of T/R-NPs. As indicated by the fluorescence, T/R-NPs were mainly located in the cytoplasm with little in the nucleus.

Quantitative analysis was conducted by a fluorescent spectrometer. The lower panel in Figure 2 shows the uptake efficiency of coumain-6-loaded T/R-NPs when incubated with cells for different time periods. It is noted that the uptake efficiency increased in a time-dependent manner during the first $2 \mathrm{~h}$. For instance, the uptake efficiency was $<40 \%$ after incubation for $30 \mathrm{~min}$, whereas it increased steadily to $\sim 70 \%$ when the incubation time was extended to $2 \mathrm{~h}$. However, $3 \mathrm{~h}$ of incubation did not increase uptake efficiency further.

A

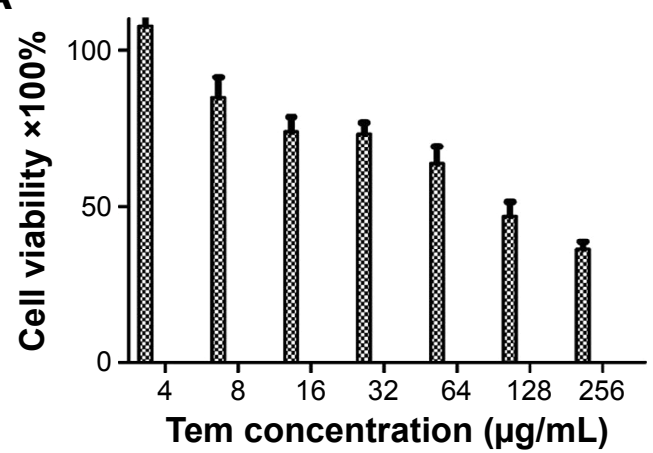

C

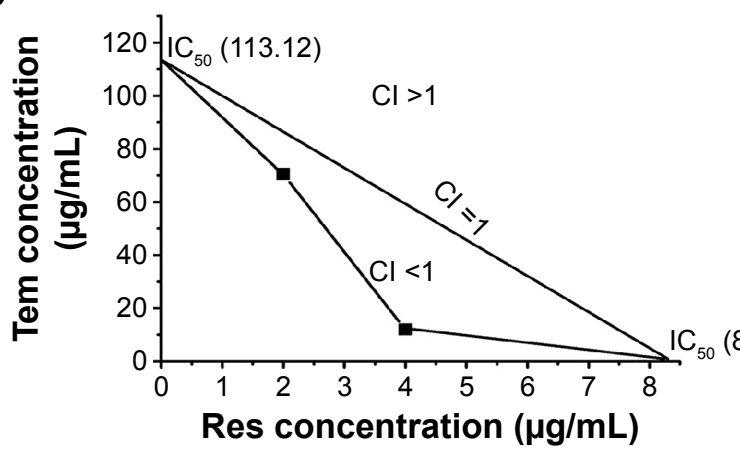

\section{Determination of in vitro cytotoxicity} and apoptotic induction

Blank nanoparticles were nontoxic to glioma cells with the highest concentration tested $(500 \mu \mathrm{g} / \mathrm{mL})$, which is much higher than the dose of T/R-NPs applied to cells (Figure S4). As shown in Figure 3A and B, both Tem and Res inhibited the growth of glioma cells in a dose-dependent manner. The half maximal inhibitory concentration values of Tem and Res were observed to be 113.3 and $8.33 \mu \mathrm{g} / \mathrm{mL}$, respectively. An isobologram analysis was conducted to evaluate the synergistic cytotoxicity of Tem and Res. The CIs were determined at two doses of Res ( 2 and $4 \mu \mathrm{g} / \mathrm{mL}$ ) combined with a series of concentration of Tem (Figure 3C). The isobologram analysis showed the CI value of 0.86 when $2 \mu \mathrm{g} / \mathrm{mL}$ Res was combined with Tem. Moreover, the combination of

B
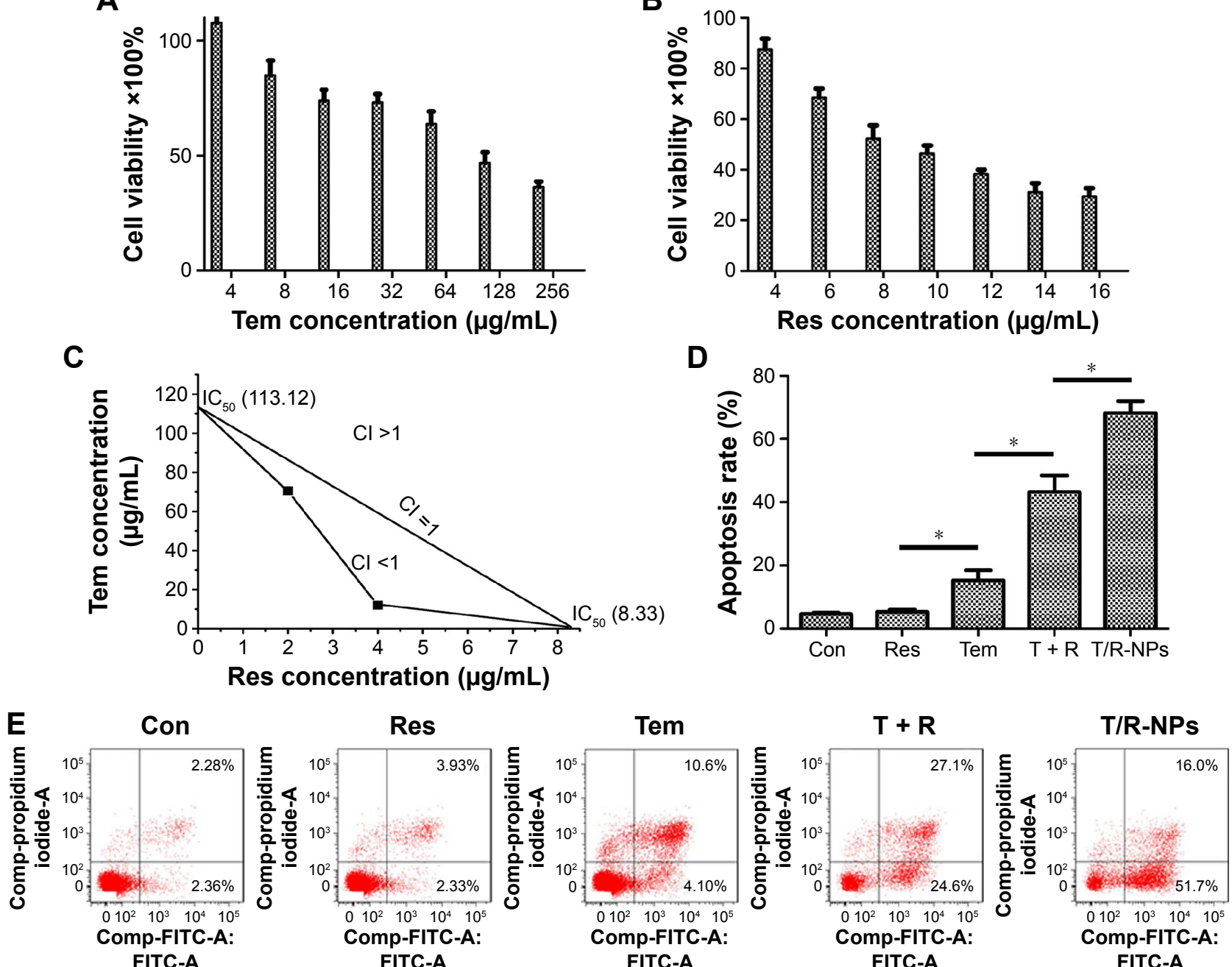

Figure 3 Cytotoxicity and apoptotic induction in $U 87$ cells.

Notes: (A) In vitro cytotoxicity of Tem on U87 cells; (B) in vitro cytotoxicity of Res on U87 cells; (C) synergistic cytotoxicity of Res and Tem by Cl; (D) apoptosis rates of U87 cells under the treatment of different agents; (E) apoptosis of U87 cells detected by FACS. $* P<0.05$ versus the corresponding group.

Abbreviations: Con, control; Cl, combination index; FACS, fluorescence-activated cell sorter; FITC, fluorescein isothiocyanate; IC, inhibitory concentration; Res, resveratrol; Tem, temozolomide; T/R-NPs, Tem/Res-coloaded nanoparticles. 
$4 \mu \mathrm{g} / \mathrm{mL}$ Res and Tem generated a lower CI value of 0.58 . Therefore, both 2 and $4 \mu \mathrm{g} / \mathrm{mL}$ Res synergistically enhanced the cytotoxicity of Tem keeping the CI values $<1$.

Apoptotic induction of T/R-NPs was determined by fluorescence-activated cell sorter (FACS; Figure 3D and E). It is noted that the apoptosis rate of glioma cells induced by T/R-NPs was $>60 \%$, which was the highest among all the groups. The equivalent dose of Tem or Res led to an apoptosis rate of $6.3 \%$ and $14.6 \%$, respectively. The combination of the two drugs resulted in an apoptosis rate of $\sim 40 \%$, which was significantly lower than that induced by T/R-NPs.

\section{Effect of T/R-NPs on the expression of related proteins}

In order to elucidate the possible mechanism underlying superior anticancer effect of T/R-NPs, Western blot was performed to evaluate the influence of drugs on related proteins. As shown in Figure 4A, a single treatment of Res $(4 \mu \mathrm{g} / \mathrm{mL})$ or Tem $(12 \mu \mathrm{g} / \mathrm{mL})$ almost had no inhibition on the phosphorylation of Akt proteins. In contrast, cotreatment of Res and Tem at the equivalent doses induced $\sim 30 \%$ reduction of p-Akt expression. Moreover, cotreatment of Res and Tem in nanoparticles (T/R-NPs) significantly decreased the expression of $\mathrm{p}$-Akt.

The expression of apoptotic proteins in U87 cells when exposed to the equivalent dose of free drugs and drug-loaded nanoparticles (Figure 4B) was further examined. T/R-NPs were the most effective to attenuate the expression of antiapoptotic protein $\mathrm{Bcl}-2$ and increase the expression of the proapoptotic protein Bax. The Bcl-2/Bax ratio was observed to be the lowest when compared to the corresponding group treated by the combination of Tem and Res at the equivalent doses, demonstrating the strongest efficiency of apoptotic induction by T/R-NPs.

\section{Effect of T/R-NPs on cell migration and invasion}

Wound healing assay was performed to evaluate the ability of migration of U87 cells. Res at the concentration of $4 \mu \mathrm{g} / \mathrm{mL}$ exhibited no obvious inhibition of the migration of U87 cells, whereas $12 \mu \mathrm{g} / \mathrm{mL}$ led to a decrease of wound healing rate of $\sim 25 \%$ (Figure 5 ). Moreover, a drop of $\sim 35 \%$ in the healing rate was achieved when U87 cells were treated with free drug combinations. Most importantly, T/R-NPs exhibited the strongest antimigration effect with a wound healing rate being only $30 \%$, which was significantly lower than the rate of the drug combinations.

\section{In vivo study of T/R-NPs in a xenograft model}

In vivo antitumor effect of T/R-NPs was evaluated by using a U87-xenograft nude mice model. Res was administered at

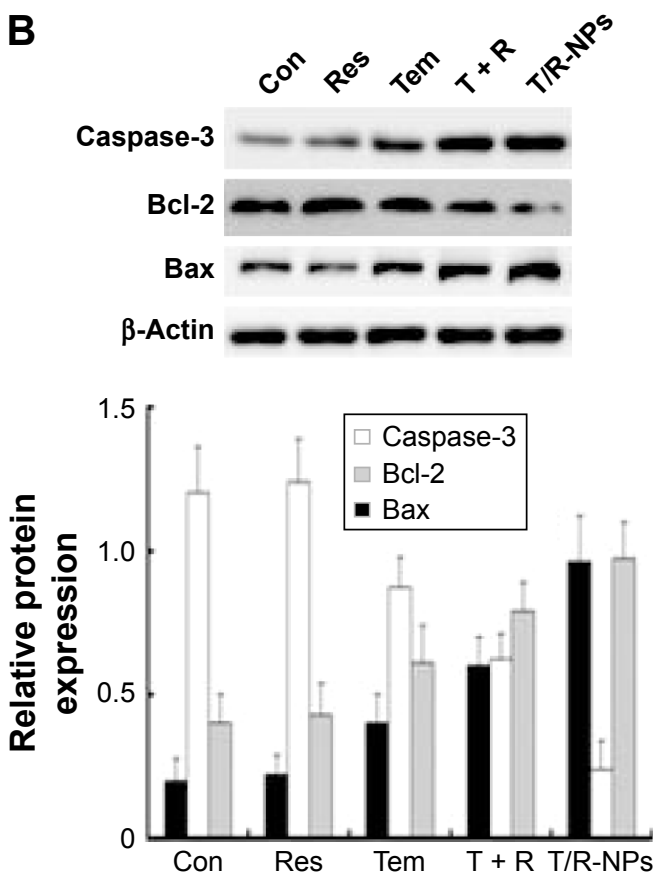

Figure 4 Protein expression of U87 cells when treated with T/R-NPs.

Notes: (A) The expression of p-Akt and Akt; (B) the expression of apoptotic proteins.

Abbreviations: Con, control; Res, resveratrol; Tem, temozolomide; T/R-NPs, Tem/Res-coloaded nanoparticles. 

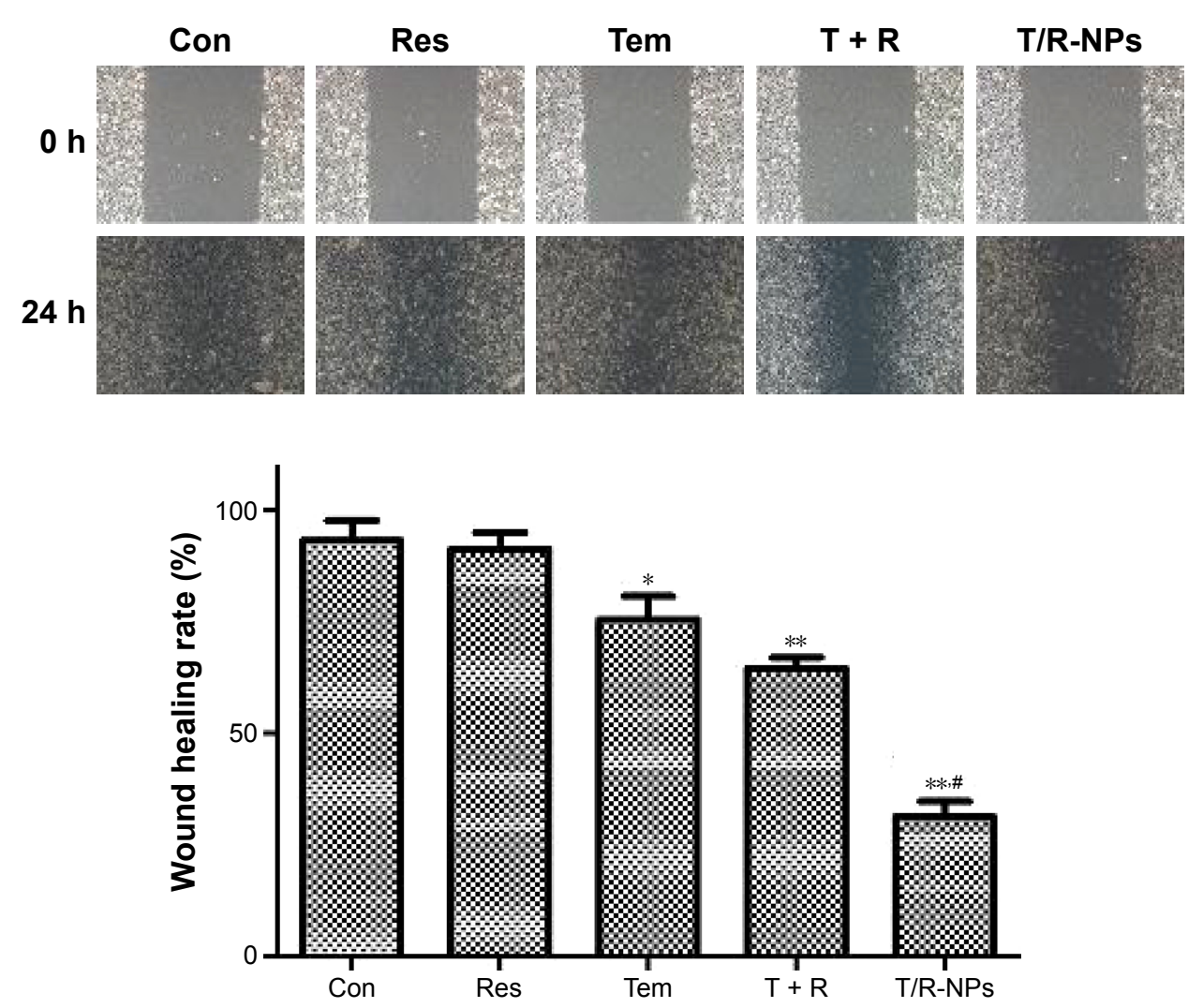

Figure 5 Wound healing assay of U87 cells when treated with T/R-NPs.

Notes: ${ }^{*} P<0.05$ versus control group; ${ }^{* * P}<0.01$ versus control group. ${ }^{*} P<0.05$ vs $T+R$ group.

Abbreviations: Con, control; Res, resveratrol; Tem, temozolomide; T/R-NPs, Tem/Res-coloaded nanoparticles; h, hours.

a lower dose of $10 \mathrm{mg} / \mathrm{kg}$, whereas Tem was injected at a dose of $30 \mathrm{mg} / \mathrm{kg}$ IP. The drug combination of Res and Tem was administered at both equivalent doses. The T/R-NPs were also injected IP at the equivalent doses. As shown in Figure 6, Res had no obvious antitumor effect with the tumor volume almost similar to the control group, whereas Tem showed moderate tumor delaying efficacy $(P=0.042$ vs control). Coadministration of Tem and Res showed a stronger effect when compared to control ( $P=0.033$ vs control). However, there was no significant difference between Tem-treated group and Tem/Res-treated group $(P=0.065)$. Most importantly, codelivery of both the drugs in nanoparticles significantly increased the anticancer efficacy than that of free drug combinations ( $P=0.033$ vs the corresponding group). In order to predict the adverse effects of the different therapy regimens, the body weight variations were analyzed. All the experimental groups showed favorable results without any significant weight loss even at the highest administered dose (Figure 6B).

\section{Discussion}

There is no curative intervention available for patients suffering from a glioma. ${ }^{26}$ Side effects and the poor survival outcome of the currently available drugs have driven researchers to look for new strategies to improve the longevity of the glioma patients. Cotreatment of Tem and Res was demonstrated to generate a significantly stronger antitumor effect than either single or combinational administration of both the drugs through CI analysis, which laid a strong foundation of the codelivery of both the drugs for glioma therapy. ${ }^{8}$ In the current study, Res was employed as a chemosensitizer to synergistically enhance the antitumor effect of Tem. However, poor solubility of Res remained a big hurdle for the potential clinical application..$^{27,28}$ The use of nanotechnology was exploited to address the issue of solubility and improve the kinetics. In the previous reports, Res-loaded nanoparticles with mPEG-PCL as drug carriers were prepared, and the superior efficacy against free Res was proved, demonstrating the advantage of nano-drug delivery systems. ${ }^{7,14,15}$ In the present study, in order to improve the solubility and achieve the better antitumor effect of combinational administration of Tem and Res, mPEG-PCL polymeric nanoparticle-based drug delivery systems were utilized to coload both the drugs. Our preliminary results showed that Tem-loaded mPEG-PCL nanoparticles were highly unstable, whereas the addition of Res significantly 
A

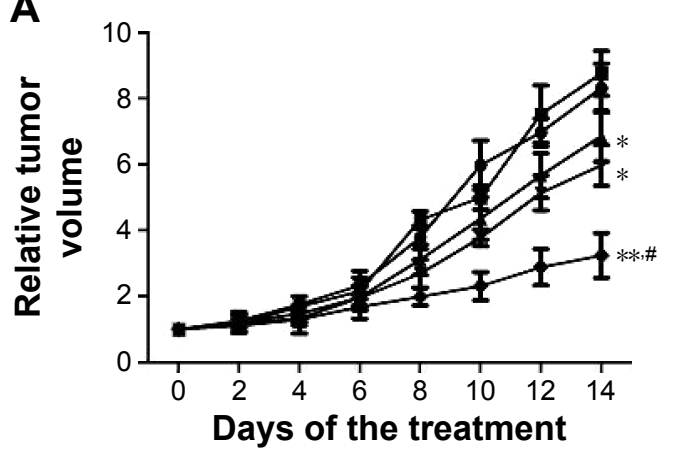

B

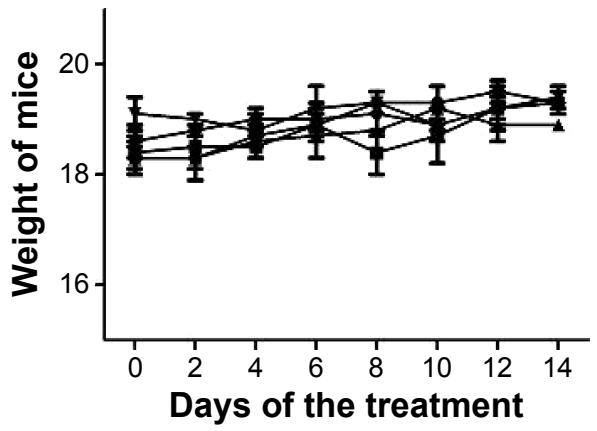

$\bullet-$ Con $\rightarrow$ Res $₫$ Tem $\rightarrow T+R \rightarrow$ T/R-NPs

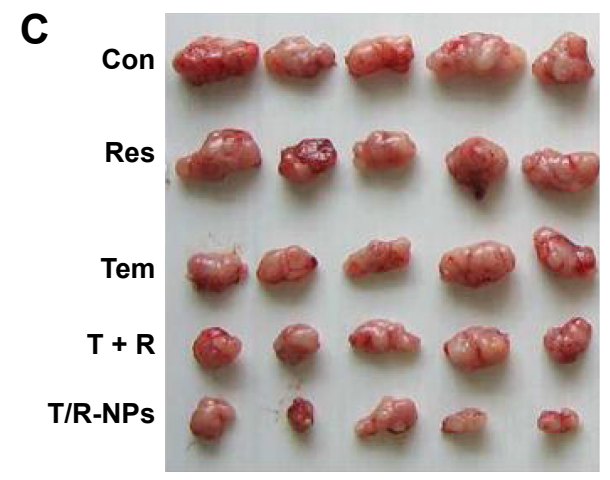

Figure 6 In vivo antitumor effect of T/R-NPs.

Notes: (A) Tumor growth curves of mice treated with different agents during the experiment; (B) change of body weight of mice during the experiment; (C) image of tumor taken from different groups. ${ }^{*} P<0.05$ versus control group; ${ }^{* * P}<0.01$ versus control group; ${ }^{*} P<0.05$ versus $T+R$ group.

Abbreviations: Con, control; Res, resveratrol; Tem, temozolomide.

increased the stability of Tem-loaded nanoparticles. This observation further strengthens the application of Res/Temcoloaded nanoparticles for the glioma tumor.

As reported in the present study, the stability of coencapsulation of Res and Tem in mPEG-PCL nanoparticles not only provided the possibility of codrug delivery through nano-drug system but also preserved the synergistic antitumor effects of both the drugs. The FACS data (Figure 3) revealed that there is significantly higher apoptotic induction in U87 cells treated with T/R-NPs than with the combination of free drugs. These results were further correlated with the inhibition of cell migratory ability in the T/R-NPs treatment group.

The possible mechanism of action underlying the synergistic effect at a molecular level was investigated. The phosphoinositide 3-kinase (PI3K)/Akt/mammalian target of rapamycin (mTOR) pathway is essential for the growth and proliferation of cancer cells, which is constitutively activated in a series of cancer cells. ${ }^{29}$ As reported in a previous study, the hyperactivation of the Akt survival pathway is important to the resistance of cancer cells including glioma. Inhibition of PI3K/Akt/mTOR pathway is effective in overcoming the therapy resistance of cancer cells. ${ }^{30,31}$ The inhibition of p-Akt expression, followed by the caspase 3-dependent apoptotic induction, was observed, which was characterized by the downregulation of antiapoptotic protein Bcl-2 and upregulation of the proapoptotic protein Bax in the T/R-NPs treatment group. These results corroborated with the previous studies where Res had been shown to enhance the cytotoxicity of Tem through AMPK-TSC-mTOR signaling pathway, leading to mitotic catastrophe and senescence. ${ }^{8,10}$ Most importantly, it was observed that drug-loaded nanoparticles were much more effective in regulating the expression of related proteins than the corresponding dose of the free drug combination, which may be attributed to the efficient uptake of nanoparticles by glioma cells. As reported previously, cellular uptake of nanoparticles was more efficient than the diffusion of free drugs. ${ }^{32}$ The present strategies were validated by using the U87-xenograft model system. In the same line, the present in vivo study demonstrated the superior tumor growth delaying the effect of T/R-NPs as compared to free drug combination or single drug treatment group alone.

\section{Conclusion}

In summary, the current study is the first demonstration of simultaneous codelivery of Res and Tem in mPEG-PCL nanoparticles with excellent antiglioma effect. Res improved the stability of Tem in the mPEG-PCL nanoparticles. The coloaded nanoparticles exhibited reinforced cytotoxicity and 
tumor delaying effect in glioma cells and xenograft models. Inhibition of the $\mathrm{p}$-Akt expression leading to tumor cell death is the plausible mechanism of action of these combination drugs. Although further study is required to investigate the probable mechanism of action of these combination drugs, however, these coloaded nanoparticles have the potential to counter the spread of malignant glioma and may shed light on the fight of glioma cells that are resistant to Tem.

\section{Acknowledgments}

This study was supported by the National Natural Science Foundation of China (Nos 81101902, 81472781, and 81572924) and the Priority Academic Program Development of Jiangsu Higher Education Institutions.

\section{Disclosure}

The authors report no conflicts of interest in this work.

\section{References}

1. Friedman HS, Kerby T, Calvert H. Temozolomide and treatment of malignant glioma. Clin Cancer Res. 2000;6(7):2585-2597.

2. Koekkoek JA, Dirven L, Heimans JJ, et al. Seizure reduction in a low-grade glioma: more than a beneficial side effect of temozolomide. J Neurol Neurosurg Psychiatry. 2015;86(4):366-373.

3. Cui X, Zhu W, Wang P, Wang X. Tetrandrine inhibits the intracellular calcium ion level and upregulates the expression of Brg1 and AHNAK in Hep-2 cells. Clin Lab. 2015;61(10):1569-1576.

4. Zulueta A, Caretti A, Signorelli P, Ghidoni R. Resveratrol: a potential challenger against gastric cancer. World J Gastroenterol. 2015;21(37): 10636-10643.

5. Xu Q, Zong L, Chen X, et al. Resveratrol in the treatment of pancreatic cancer. Ann N Y Acad Sci. 2015;1348(1):10-19.

6. Filippi-Chiela EC, Villodre ES, Zamin LL, Lenz G. Autophagy interplay with apoptosis and cell cycle regulation in the growth inhibiting effect of resveratrol in glioma cells. PLoS One. 2011;6(6):e20849.

7. Shao J, Li X, Lu X, et al. Enhanced growth inhibition effect of resveratrol incorporated into biodegradable nanoparticles against glioma cells is mediated by the induction of intracellular reactive oxygen species levels. Colloids Surf Biointerfaces. 2009;72(1):40-47.

8. Yuan Y, Xue X, Guo RB, Sun XL, Hu G. Resveratrol enhances the antitumor effects of temozolomide in glioblastoma via ROS-dependent AMPK-TSC-mTOR signaling pathway. CNS Neurosci Ther. 2012; 18(7):536-546.

9. Li H, Liu Y, Jiao Y, et al. Resveratrol sensitizes glioblastoma-initiating cells to temozolomide by inducing cell apoptosis and promoting differentiation. Oncol Rep. 2015;35(1):343-351.

10. Filippi-Chiela EC, Thome MP, Bueno e Silva MM, et al. Resveratrol abrogates the temozolomide-induced G2 arrest leading to mitotic catastrophe and reinforces the temozolomide-induced senescence in glioma cells. BMC Cancer. 2013;13:147.

11. Huang H, Lin H, Zhang X, Li J. Resveratrol reverses temozolomide resistance by downregulation of MGMT in T98G glioblastoma cells by the NF-kappaB-dependent pathway. Oncol Rep. 2012;27(6):2050-2056.

12. Lin CJ, Lee CC, Shih YL, et al. Resveratrol enhances the therapeutic effect of temozolomide against malignant glioma in vitro and in vivo by inhibiting autophagy. Free Radic Biol Med. 2012;52(2):377-391.

13. Fuggetta MP, D'Atri S, Lanzilli G, et al. In vitro antitumour activity of resveratrol in human melanoma cells sensitive or resistant to temozolomide. Melanoma Res. 2004;14(3):189-196.
14. Lu X, Xu H, Sun B, Zhu Z, Zheng D, Li X. Enhanced neuroprotective effects of resveratrol delivered by nanoparticles on hydrogen peroxide-induced oxidative stress in rat cortical cell culture. Mol Pharm. 2013;10(5):2045-2053.

15. Yin $\mathrm{H}, \mathrm{Si}$ J, Xu H, et al. Resveratrol-loaded nanoparticles reduce oxidative stress induced by radiation or amyloid-beta in transgenic Caenorhabditis elegans. J Biomed Nanotechnol. 2014;10(8):1536-1544.

16. Teskac K, Kristl J. The evidence for solid lipid nanoparticles mediated cell uptake of resveratrol. Int J Pharm. 2010;390(1):61-69.

17. Fang C, Wang K, Stephen ZR, et al. Temozolomide nanoparticles for targeted glioblastoma therapy. ACS Appl Mater Interfaces. 2015;7(12): 6674-6682.

18. Xu H, Hou Z, Zhang H, et al. An efficient Trojan delivery of tetrandrine by poly(N-vinylpyrrolidone)-block-poly(epsilon-caprolactone) (PVP-bPCL) nanoparticles shows enhanced apoptotic induction of lung cancer cells and inhibition of its migration and invasion. Int J Nanomedicine. 2014;9:231-242.

19. Li X, Lu X, Xu H, et al. Paclitaxel/tetrandrine coloaded nanoparticles effectively promote the apoptosis of gastric cancer cells based on "oxidation therapy". Mol Pharm. 2012;9(2):222-229.

20. Li X, Zhen D, Lu X, et al. Enhanced cytotoxicity and activation of ROS-dependent c-Jun NH2-terminal kinase and caspase-3 by low doses of tetrandrine-loaded nanoparticles in Lovo cells - a possible Trojan strategy against cancer. Euro J Pharm Biopharm. 2010;75(3): $334-340$.

21. Li X, Li R, Qian X, et al. Superior antitumor efficiency of cisplatinloaded nanoparticles by intratumoral delivery with decreased tumor metabolism rate. Euro J Pharm Biopharm. 2008;70(3):726-734.

22. Zhang H, Li X, Ding J, et al. Delivery of ursolic acid (UA) in polymeric nanoparticles effectively promotes the apoptosis of gastric cancer cells through enhanced inhibition of cyclooxygenase 2 (COX-2). Int J Pharm. 2013;441(1-2):261-268.

23. Jiang T, Li Y, Lv Y, Cheng Y, He F, Zhuo R. Biodegradable amphiphilic block-graft copolymers based on methoxy poly(ethylene glycol)-b-(polycarbonates-g-polycarbonates) for controlled release of doxorubicin. J Mater Sci Mater Med. 2014;25(1):131-139.

24. Jain A, Jain SK. Formulation and optimization of temozolomide nanoparticles by 3 factor 2 level factorial design. Biomatter. 2013;3(2): pii:e25102.

25. Chou TC, Talalay P. Quantitative analysis of dose-effect relationships: the combined effects of multiple drugs or enzyme inhibitors. $A d v$ Enzyme Regul. 1984;22:27-55.

26. Berrocal A, Gil M, Gallego O, et al. SEOM guideline for the treatment of malignant glioma. Clin Transl Oncol. 2012;14(7):545-550.

27. Sanna V, Siddiqui IA, Sechi M, Mukhtar H. Resveratrol-loaded nanoparticles based on poly(epsilon-caprolactone) and poly(D,L-lactic-coglycolic acid)-poly(ethylene glycol) blend for prostate cancer treatment. Mol Pharma. 2013;10(10):3871-3881.

28. Jung KH, Lee JH, Park JW, et al. Resveratrol-loaded polymeric nanoparticles suppress glucose metabolism and tumor growth in vitro and in vivo. Int J Pharm. 2015;478(1):251-257.

29. Liang J, Slingerland JM. Multiple roles of the PI3K/PKB (Akt) pathway in cell cycle progression. Cell Cycle. 2003;2(4):339-345.

30. Dai C, Zhang B, Liu X, et al. Inhibition of PI3K/AKT/mTOR pathway enhances temozolomide-induced cytotoxicity in pituitary adenoma cell lines in vitro and xenografted pituitary adenoma in female nude mice. Endocrinology. 2013;154(3):1247-1259.

31. Niessner H, Forschner A, Klumpp B, et al. Targeting hyperactivation of the AKT survival pathway to overcome therapy resistance of melanoma brain metastases. Cancer Med. 2013;2(1):76-85.

32. Davda J, Labhasetwar V. Characterization of nanoparticle uptake by endothelial cells. Int J Pharm. 2002;233(1-2):51-59. 


\section{Supplementary materials}

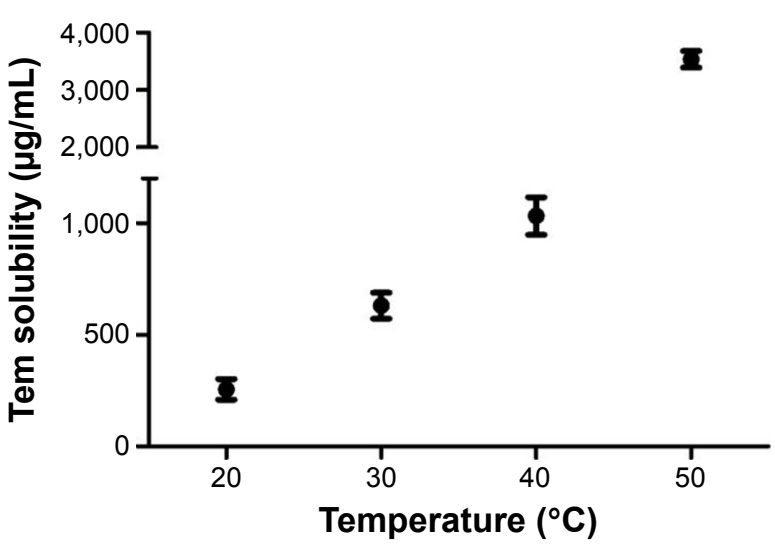

Figure SI The solubility data of Tem at different temperatures. Abbreviation: Tem, temozolomide.

Res

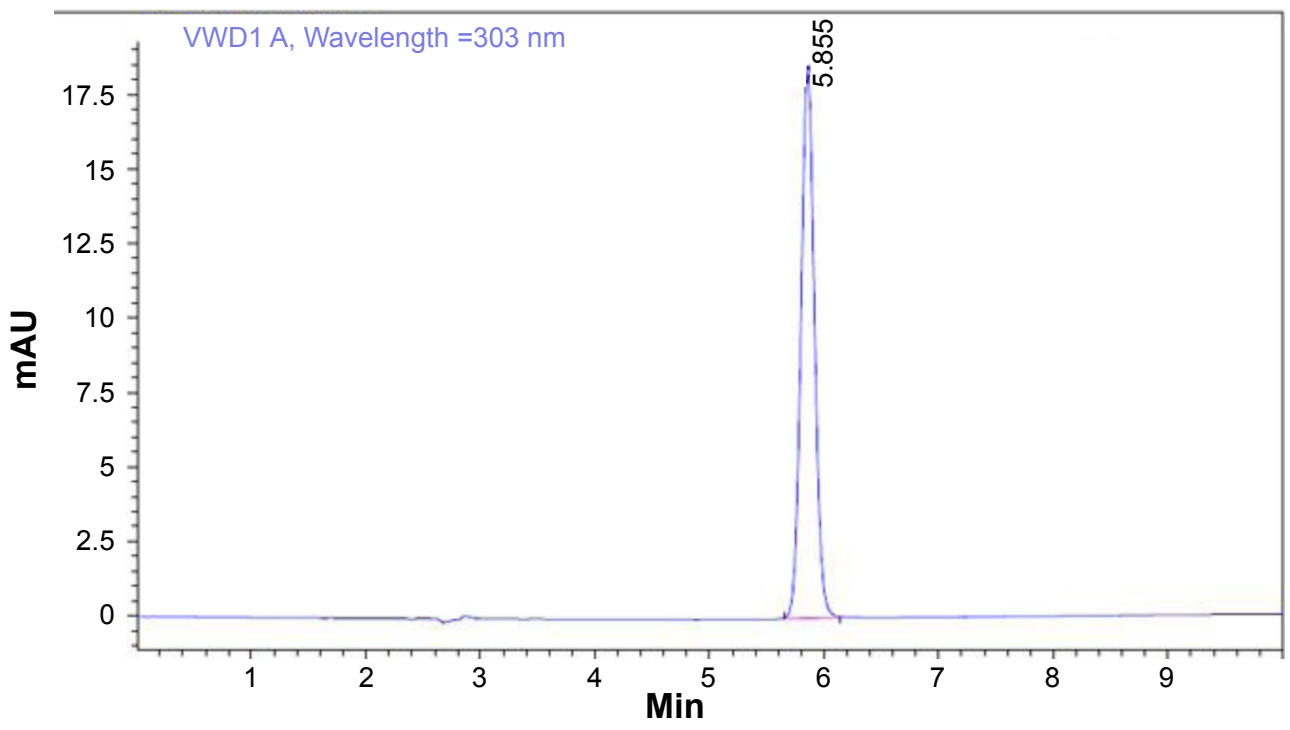

Tem

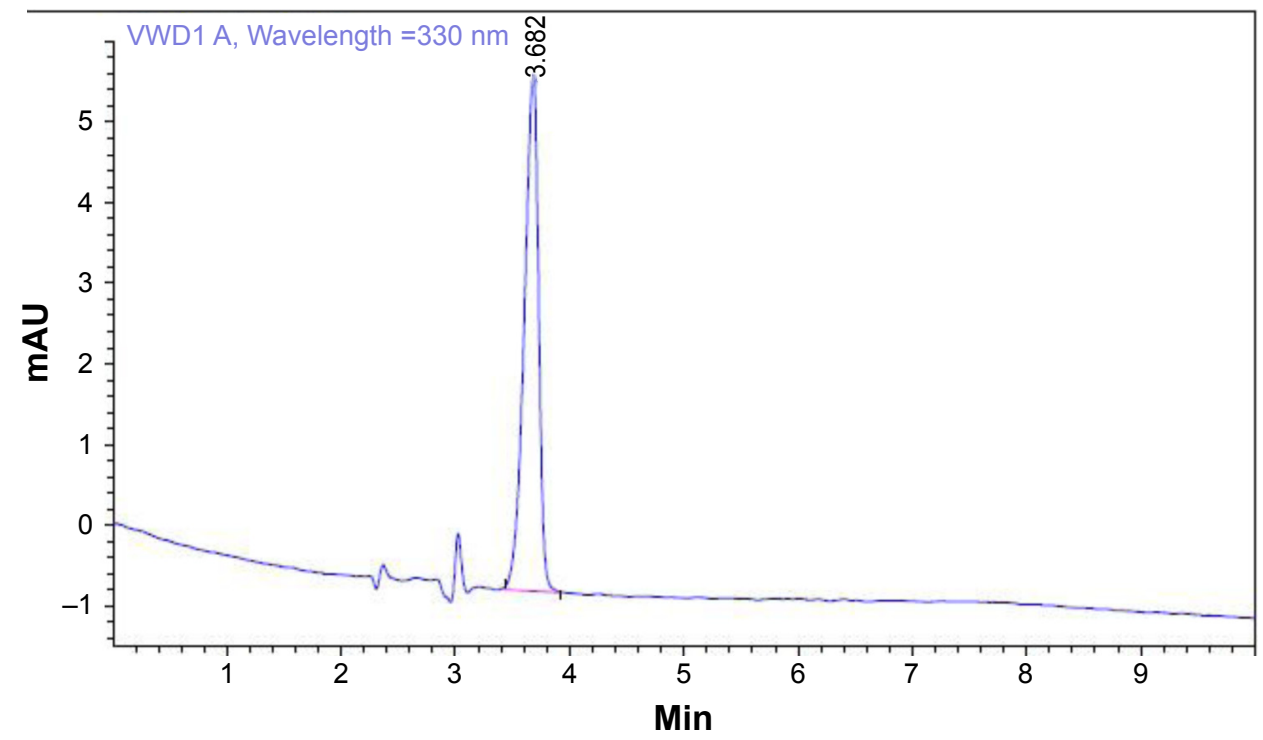

Figure S2 HPLC data of Res and Tem.

Abbreviations: HPLC, high-performance liquid chromatography; Res, resveratrol; Tem, temozolomide. 


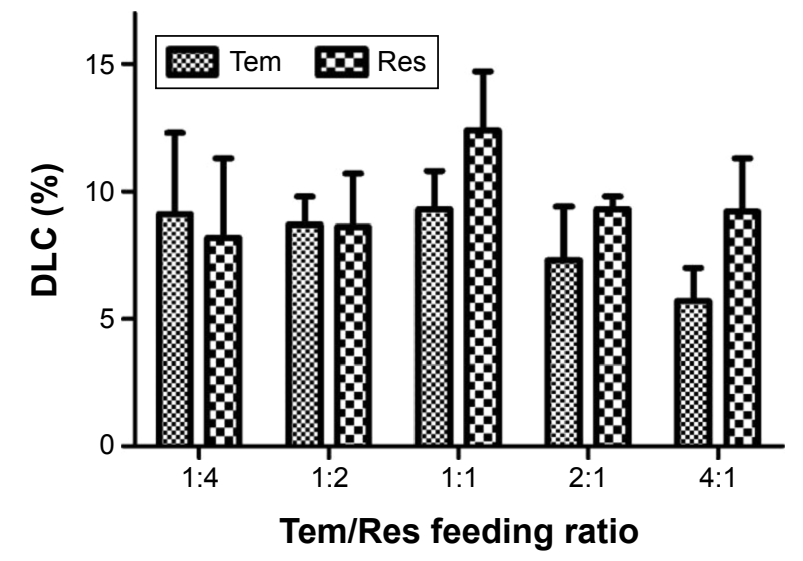

Figure S3 DLC of Tem and Res at different feeding ratios.

Abbreviations: DLC, drug loading content; HPLC, high-performance liquid chromatography; Res, resveratrol; Tem, temozolomide.

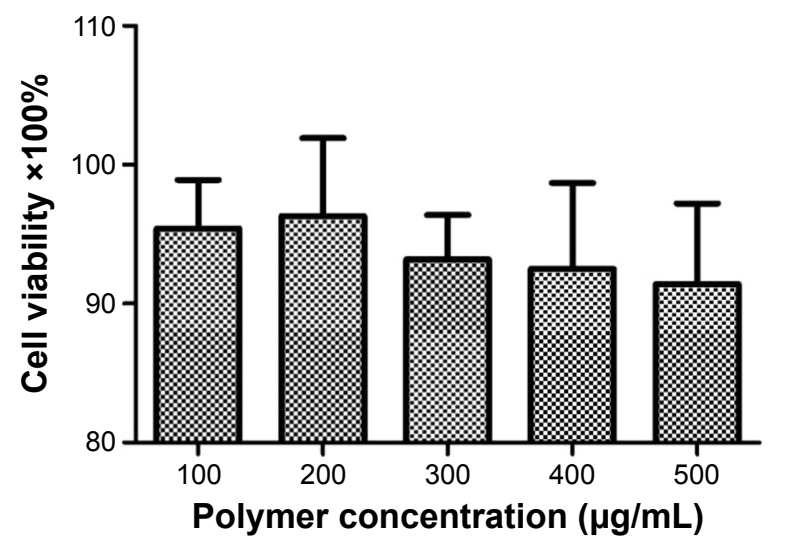

Figure S4 Cytotoxicity of blank nanoparticles at different polymer concentration.

\section{Publish your work in this journal}

The International Journal of Nanomedicine is an international, peerreviewed journal focusing on the application of nanotechnology in diagnostics, therapeutics, and drug delivery systems throughout the biomedical field. This journal is indexed on PubMed Central, MedLine, CAS, SciSearch $®$, Current Contents ${ }^{\circledR} /$ Clinical Medicine,
Journal Citation Reports/Science Edition, EMBase, Scopus and the Elsevier Bibliographic databases. The manuscript management system is completely online and includes a very quick and fair peer-review system, which is all easy to use. Visit http://www.dovepress.com/ testimonials.php to read real quotes from published authors. 